\title{
Programa para la mejora de la empleabilidad de los egresa- dos en traducción y interpretación. Un estudio de caso
}

Anabel Galán-Mañas ${ }^{1}$

Resumo: O objetivo deste trabalho é analisar o conteúdo do programa para a melhoria da empregabilidade dos egressos em tradução e interpretação, implementado na Universitate Autònoma de Barcelona entre os cursos 20112012 e 2014-2015. A finalidade do programa foi melhorar a inserção laboral dos diplomados, não só para aumentar as chances de empregabilidade, que entre os nossos diplomados é muito alta, mas também para que eles consigam obter um posto e uma remuneração adequados para os estudos realizados. Para alcançar essa finalidade, foram concebidas intervenções de vários tipos a fim de que os estudantes: conheçam o funcionamento do mercado de trabalho - especialidades, workflow, gestão contábil e fiscal -; tomem consciência de suas próprias habilidades; construam os documentos necessários para a procura ativa de emprego - portfólio profissional, currículo e carta de motivação -; possam optar pela via do empreendedorismo por estar familiarizados com a construção de um profissional ou um projeto comercial.

As ações realizadas consistiram em: palestras, oficinas, visitas a empresas, práticas curriculares, preparação do trabalho de fim de estudos relacionado com um projeto empreendedor, aulas práticas, realização de um teste de competências, divulgação de ofertas de trabalho e tutorias personalizadas.

Palavras-chave: Empregabilidade; inserção laboral; empreendedorismo; egressos em tradução e interpretação.

Resumen: El objetivo de este artículo es analizar el contenido del programa para la mejora de la empleabilidad para los egresados en traducción e interpretación llevado a cabo en a Universitat Autònoma de Barcelona del curso 2011-2012 al 2014-2015. La finalidad del programa fue mejorar la inserción de los egresados, no solo para aumentar las posibilidades de inserción laboral, que entre nuestros egresados es muy alta, sino para conseguir que los puestos que ocupen y el nivel de ingresos sea adecuado con los estudios cursados.

Para alcanzarlo, se diseñaron intervenciones de diversa índole que promovieron que el estudiantado: conociera el funcionamiento del mercado de trabajo - especialidades, flujo de trabajo, gestión contable y fiscal-; tomara conciencia de sus propias capacidades; construyera los documentos necesarios para la búsqueda activa de empleo - carpeta profesional, currículo, carta de motivación-; pudiera optar por la vía del emprendimiento al estar familiarizado con la construcción de un proyecto profesional o empresarial. Las acciones llevadas a cabo consistieron en: conferencias, talleres, visitas a entidades, prácticas curriculares, elaboración del trabajo de fin de grado relacionado con un proyecto emprendedor, clases prácticas, realización de un

1 Departament de Traducció i d'Interpretació i d'Estudis de l'Àsia Oriental. Universitat Autònoma de Barcelona. 
test de competencias, difusión de ofertas de trabajo y tutorías personalizadas.

Palabras clave: Empleabilidad; inserción laboral; emprendimiento; egresados en traducción e interpretación.

\section{Introducción}

A menudo se ha criticado a la universidad de la falta de correspondencia entre la formación que ofrece y las necesidades del mercado de la traducción y la interpretación (Li 2001, 2002; Bowker, 2004; Schellekens, 2004; Gouadec, 2007). Algunos autores (Bowker, 2004, Schellekens, 2004) atribuyen este desajuste a una formación demasiado académica y que no prepara a los estudiantes para la profesión, de ahí las dificultades de los egresados a acceder a un empleo satisfactorio.

Según los resultados de un estudio de mercado llevado a cabo recientemente con egresados de 2004/05 a 2013/14 del grado y la licenciatura en traducción e interpretación de la Universitat Autònoma de Barcelona (Galán-Mañas, 2017), el 90\% de los egresados está trabajando. Este dato confirma lo que apuntan Chouc y Calvo (2010), que sostienen que la inserción laboral de los egresados en traducción e interpretación es muy alta si la comparamos con otros estudios, tanto en España como en el extranjero.

Según ese mismo estudio, un 35\% de los egresados se dedica a tareas que no son estrictamente de traducción o interpretación, y que tienen que ver con el comercio exterior, el secretariado, la organización de eventos, la hostelería, el turismo, etc. Esto significa que, a pesar de que el grado de inserción de los egresados en traducción interpretación en el mercado es alto, su empleo no siempre es satisfactorio porque no tiene una relación directa con los estudios.

En el actual Espacio Europeo de Educación Superior (EEES), dos de los pilares son la empleabilidad y la formación por competencias. Según el Proceso de Bolonia 2020, puesto que el mercado laboral requiere niveles de capacidad y competencias transversales cada vez mayores, la educación superior debe dotar a los alumnos de las necesarias habilidades y competencias y los conocimientos avanzados a lo largo de toda su vida profesional, de modo que el individuo pueda aprovechar plenamente las oportunidades del cambiante mercado laboral.

Para que las instituciones de educación superior alcancen este objetivo, el EEES las insta a mejorar la provisión, accesibilidad y calidad de sus servicios de orientación profesional y de empleo para alumnos y graduados, y a incorporar el empleo a los programas de estudio, así como el aprendizaje en el puesto de trabajo.

Siguiendo las instrucciones del EEES, el Estatuto del Estudiante Universitario (Ministerio de Educación de España, 2010) incluye el derecho de los estudiantes del grado a recibir orientación y tutoría personalizadas en la fase final con el objetivo de facilitar la incorporación laboral y el desarrollo profesional. Ello implicará incluir itinerarios formativos y salidas profesionales, formación en competencias transversales y el diseño del proyecto profesional para facilitar la empleabilidad y la incorporación laboral.

De la formulación del Estatuto del Estudiante Universitario se desprende que la empleabilidad y la incorporación laboral son conceptos distintos. La inserción laboral representa el punto de conjunción entre la empleabilidad —entendida como la probabilidad de inserción que depende del individuo-, y la ocupabilidad —entendida como la capacidad de inserción que depende del mercado de trabajo- (Bisquerra, 1992a). La inserción laboral hace referencia a encontrar un empleo, mientras que la empleabilidad agrupa en- 
contrar empleo y mantenerlo (Hillage y Pollard, 1998). Según García y Pérez (2008), esta diferencia lleva a la necesidad de una capacitación estructural, y no coyuntural.

La universidad puede incidir en la empleabilidad de sus egresados, si bien un conocimiento exhaustivo del mercado le permitiría adaptar su plan de estudios a las necesidades del mercado, y facilitar así también su ocupabilidad. A pesar de la importancia de conocer el mercado para mejorar los planes de estudio, como apuntan Kelly (2003) y Calvo (2006), los estudios del mercado de la traducción y la interpretación en España son todavía escasos: Agencia Nacional de Evaluación de la Calidad y Acreditación, conocida como ANECA, (2005), Agrupación de centros especializados en traducción (Orf, 2005).

En España, de acuerdo con el Real Decreto 1393/2007 de 29 de octubre, "las enseñanzas de grado tienen como finalidad la obtención por parte del estudiante de una formación general junto con otras orientadas a la preparación para el ejercicio de actividades de carácter profesional", de modo que esta "nueva organización de las enseñanzas incrementará la empleabilidad de los titulados". De ello se desprende, tal y como defiende el Instituto de Ciencias de la Educación, ICE, (2008), que la finalidad de los estudios universitarios es la preparación profesional y no la preparación académica, haciendo referencia a una formación que no solo contemple las competencias específicas, sino también las transversales.

Ahora bien, ¿qué hace que una persona sea empleable? Como asegura Calvo (2013), en los mercados de trabajo más «sanos», empleabilidad e inserción laboral pueden ir de la mano con facilidad, porque los egresados realmente encuentran trabajos acordes a sus capacidades. Sin embargo, en un momento de gran competencia en el mercado de trabajo, una titulación no asegura por sí misma el éxito profesional. Las empresas, ante egresados con la misma titulación, valoran a los titulados que posean ciertas competencias que garanticen un mayor rendimiento en el puesto de trabajo.

La formación para la empleabilidad debería abarcar desde el inicio de su formación hasta, como mínimo, su graduación y, como sugiere Romero (2004) debería contemplar: la formación académica (que implicará una filosofía de aprendizajes activos y aplicados), la experiencia laboral, los conocimientos de inserción, las técnicas de búsqueda de puestos vacantes y las competencias.

Desde nuestro punto de vista, cada centro debería disponer de un programa de formación para mejorar la empleabilidad de sus estudiantes, a parte de los servicios de ocupación que pueda tener la universidad. El hecho de que el programa se diseñe en el propio centro garantizará una formación que contemple las especificidades de la profesión.

El objetivo de este artículo es analizar algunas de las acciones que se pueden llevar a cabo para mejorar la empleabilidad de los egresados en traducción e interpretación. Se trata de un primer balance del programa para la mejora de la empleabilidad implementado en la Facultad de Traducción e Interpretación de la Universitat Autònoma de Barcelona.

\section{La empleabilidad en la formación de Traductores}

En el ámbito de la traducción y la interpretación, si bien en el mapa de la disciplina que dibujó Holmes (1972 y 1988) ni siquiera se contemplaba el ámbito laboral como objeto de estudio, en la actualidad existe un creciente interés por todo lo "profesional". Este interés, como señala Kuznik $(2007,2010)$ ha contribuido a que los aspectos profesionales y el ámbito laboral de los traductores e intérpretes constituyan un subcampo de estudios en Traductología, a pesar de la falta de claridad y delimitación conceptual en cuanto al adjetivo "profesional" aplicado a nuestro campo de estudio. 
En este sentido, cada vez existen más investigaciones relacionadas con distintos aspectos del mercado y el ejercicio de la profesión en un ámbito concreto de la traducción o la interpretación: la traducción técnica (Jiménez, 2002); la traducción jurídica (Monzó, 2002); la traducción audiovisual (Díaz Cintas, 2003; Chaume, 2013; Cerezo 2012 y Collet 2016); la intermediación lingüística o consultoría de gestión intercultural (Florido, 1995); la revisión (Ko, 2011); la traducción colaborativa (Klimkowski, 2006; Kageura et al 2011; Kelly, Ray, De Palma 2011); la interpretación jurada (Ortega, 2011); los traductores en las instituciones internacionales (Dam y Zethsen, 2012; Wagner, Bench and Martinez, 2002); la interpretación en los servicios públicos (Mikkelson, 2007; Morris, 2010; Sales y Valero, 2006; Angelelli, 2010); los servicios públicos (Valero, 2003); la traducción literaria (Amit-Kochavi, 2010); la traducción para agencias de noticias (Bielsa, 2007); nuevos perfiles profesionales: Aguessim (2008) trata sobre la mediación y Ahmed (2007), Badia (2006), Chrupała (2003), Del Pozo (2016) tratan sobre la tecnología que exige el mercado; el contenido de los puestos de trabajo en traducción (Kuznik (2011).

No obstante, aún son pocos los estudios que tratan sobre la intersección entre estudiantes o recién egresados y empresas. Gouadec (2007), Samuelsson-Brown (1998), Polley y Barber (1989) y (Sofer, 2004) se centran en cómo conseguir trabajo en el mercado de la traducción en general. Teichman (2003) da consejos para dar una buena impresión a los clientes y desarrollar habilidades para encontrar un trabajo en el mundo de la traducción. Ulrych (1996), Wakabayashi (2002) y Alcina (2003) tratan de la necesidad de poner en contacto a los estudiantes con profesionales para que aprendan a relacionarse y a conocer la profesión de cerca. Wagner (2002), Cámara (2004), Torres-Hostench (2013), Pym et. al (2014), Kuznik (2010) y Galán-Mañas (2014b) tratan sobre las prácticas curriculares desde distintas perspectivas. Galán-Mañas (2017) analiza la relación entre las plazas ofrecidas a los estudiantes de prácticas y las tareas que realizan los egresados. Mayr et al. (2008), Pircher et al. (2005), Lebtahi e Ibert (2004) y Ordóñez (2010), sobre las expectativas profesionales. Mayor Serrano (2005) sobre la necesidad de tener formación sobre el ámbito laboral que les permita una mejor inserción. Way (2008) presenta tareas prácticas para el aula de traducción de modo que los estudiantes sistematicen el método de trabajo profesional realizando distintas funciones. Aguilar (2004), cómo ha sido su incorporación al mercado y si sus compañeros de estudios estaban preparados para ello.

En lo que se refiere a la formación para la empleabilidad y la inserción laboral, encontramos los siguientes trabajos. Calvo (2006) hace un recorrido por los (pocos) estudios sobre la inserción laboral de los egresados en traducción e interpretación y sugiere que en algunas de las asignaturas del grado se incluyan contenidos para promover la empleabilidad de los estudiantes. Calvo y Morón (2009) analizan algunos instrumentos diseñados para desarrollar la acción tutorial para la empleabilidad en el grado de traducción e interpretación de la Universidad de Granada; estos instrumentos son: fichas de evolución personal, entrevistas semiestructuradas y cuestionarios. Torres-Hostench (2007 y 2010) presenta un programa para la orientación laboral de traductores a nivel de posgrado. Chouc y Calvo (2010) defienden la inclusión de los aspectos relacionados con la empleabilidad en el propio plan de estudios. Y Calvo et al. (2010) presentan un proyecto que consistió en un programa para la orientación laboral de estudiantes de traducción de cuarto curso.

Que existan tan pocas propuestas sobre esta temática es significativo si tenemos en cuenta que los estudiantes y los egresados a menudo consideran que no tienen suficiente formación en aspectos relacionados con la inserción laboral. 


\section{Programa para la empleabilidad en la formación de Traductores}

Siguiendo las directrices del Estatuto del Estudiante Universitario, la Facultad de Traducción e Interpretación de la UAB diseñó un programa para la mejora de la empleabilidad de los estudiantes de traducción e interpretación.

\section{OBJETIVO}

El objetivo global del programa fue mejorar la empleabilidad de los egresados en Traducción e Interpretación de la Universitat Autònoma de Barcelona. Se entendió la empleabilidad como la capacidad del individuo de acceder a un empleo adecuado para la formación recibida que permita tener unos ingresos acordes con el nivel de formación, mantenerse en el empleo y encontrar otro si fuera necesario.

Las acciones contempladas en el programa se dirigieron a:

- Tomar conciencia de la diversidad de salidas profesionales del mercado de la traducción y la interpretación.

- Jugar diferentes roles en los flujos de trabajo de un encargo de traducción e interpretación.

- $\quad$ Explorar las principales estrategias de búsqueda de empleo.

- Identificar las obligaciones contables y fiscales a que debe hacer frente el traductor o el intérprete autónomo.

- Reconocer las principales demandas del mercado de la traducción.

- Recoger datos necesarios para tomar decisiones de carrera.

- Analizar vías para el emprendimiento.

- Examinar los intereses y las competencias transversales para incrementar el autoconocimiento.

\section{DESARROLLO E IMPLEMENTACIÓN}

El programa para la inserción laboral de los graduados en traducción e interpretación corrió a cargo del vicedecanato de profesionalización, de la Facultad de Traducción e Interpretación de la Universitat Autònoma de Barcelona. Se llevó a cabo durante los cursos académicos 2011/12 y 2014/15.

\section{a. Destinatarios y agentes implicados}

Los destinatarios del programa fueron los estudiantes de Traducción e Interpretación de la Universitat Autònoma de Barcelona, tanto del grado en Traducción e Interpretación como del máster en Traducción, Interpretación y Estudios Interculturales.

La participación en el programa fue voluntaria.

Los agentes implicados en el programa fueron:

- Vicedecana de profesionalización.

- Coordinadora de las prácticas curriculares.

- Treball Campus. Se trata de la oficina de empleo de la universidad, que organiza talleres de formación y tiene una unidad para el asesoramiento e incubación de ideas de negocio. Se contó con su colaboración para la organización de algunos talleres.

- Empresas y entidades que trabajan con traducción, interpretación, mediación, revisión, etc. 


\section{Conexão Letras}

- Asociaciones de traductores e intérpretes.

- Antiguos estudiantes que trabajan en el ámbito de la traducción o la interpretación.

- Traductores e intérpretes en activo.

\section{b. Recursos económicos y personales}

Para el diseño y la implementación del programa, la Facultad de Traducción e Interpretación no dispuso de ningún tipo de recurso económico. Por esta razón, se tuvo que contar con profesionales que aceptaron participar de forma gratuita en el programa. En lo que se refiere a recursos tecnológicos e infraestructuras, se contó con el apoyo de la facultad.

\section{c. Acciones}

Las acciones que articularon el programa fueron: conferencias, talleres, visitas a entidades, consultorías personalizadas, prácticas curriculares, trabajos de fin de grado, clases prácticas de asignaturas del plan de estudios, test de competencias, difusión de ofertas de trabajo.

\section{Conferencias}

El objetivo de las conferencias fue:

- Poner en contacto a los estudiantes con los profesionales de la traducción y la interpretación.

- Dar a conocer distintas salidas profesionales.

- $\quad$ Poner en contacto al estudiantado con las asociaciones de traductores.

Las conferencias se estructuraron en una presentación de 45 o 60 minutos por parte del profesional invitado y 60 o 75 minutos de debate entre el conferenciante y los estudiantes, durante el que estos podían plantear dudas y preguntas.

Los temas tratados en las conferencias fueron variados:

- $\quad$ los inicios en la profesión;

- $\quad$ las asociaciones de traductores y los servicios a los socios;

- la agencia de traducción: gestión, búsqueda de colaboradores y búsqueda de clientes;

- la profesión del intérprete;

- la traducción jurada;

- los perfiles profesionales de la traducción o la interpretación: traducción jurada, traducción literaria, traducción audiovisual, intérprete telefónico, lingüista forense, intérprete en instituciones internacionales, corrector lingüístico, etc.

La importancia de las conferencias reside en que aquellos estudiantes que ya saben en qué se quieren especializar pueden hacer preguntas concretas sobre la especialización, si es necesario un máster para poderse especializar, etc. Para los estudiantes que no saben en qué quieren trabajar, las conferencias son útiles porque les dan un panorama de las distintas posibilidades que tienen.

\section{Talleres}

El objetivo de cada taller fue proporcionar al estudiantado las herramientas para la elaboración de documentos para la búsqueda activa de empleo. 
Se trató de sesiones prácticas en que el estudiantado tuvo que realizar distintas tareas a lo largo del taller, o después del taller. Hemos agrupado en bloques los principales temas tratados:

- Búsqueda activa de empleo para la plantilla de una empresa:

- el currículo vitae

- la carta de motivación

- la entrevista

- Inicio de la actividad profesional como autónomo:

- cómo darse de alta como autónomo

- búsqueda activa de empleo y autocandidatura

- la página web del traductor

- características de la prueba de traducción

- Diseño de un proyecto profesional o empresarial:

- $\quad$ servicio que se desea vender

- $\quad$ segmento del mercado al que se va a dirigir el servicio

- $\quad$ estrategia de desarrollo

- aspectos económicos

- $\quad$ aspectos jurídicos

- Gestión financiera de su actividad laboral:

- $\quad$ elaboración de presupuestos y facturas

- obligaciones fiscales, como las declaraciones trimestrales y anuales de IVA e IRPF

- contratos de traducción

Con los talleres del primer bloque, el estudiantado tuvo la oportunidad de elaborar su currículo vitae y la carta de motivación. Estos dos documentos fueron revisados por el docente. En la elaboración del currículo se insistió en la importancia de destacar los servicios que podrían prestar y en qué combinaciones lingüísticas. Para la elaboración de la carta de motivación, los estudiantes tuvieron que hacer un test de competencias, que les ayudó a conocerse a sí mismos para luego destacar aquellas aptitudes que podrían estar bien valoradas por las empresas. Por último, se practicó la entrevista con posibles preguntas (y respuestas) y mediante la técnica del juego de roles. Hay que tener en cuenta que la entrevista es la principal estrategia de selección que utilizan las empresas, además de la prueba de traducción, obviamente.

El segundo bloque consistió en tratar los aspectos necesarios para iniciarse en la actividad profesional, no solo cuáles son los trámites necesarios para darse de alta como autónomo en España, sino también cómo buscar empleo a través de portales de traducción e interpretación y redes sociales, cómo hacerse visible como profesional y cómo construir una marca personal. Por último, este bloque trató las características que debería tener la prueba de traducción. Este aspecto es bastante importante, ya que en el mercado de la traducción español se han dado varios casos de pruebas de traducción abusivas con las que algunas agencias de traducción pretendían conseguir la traducción de libros enteros a través de pruebas de traducción ficticias.

En el tercer bloque se quiso promover el espíritu emprendedor del estudiantado. Estos talleres consistieron en presentarles los aspectos que deberían tener en cuenta a la hora de diseñar su propio proyecto profesional o empresarial.

El bloque relativo a la gestión financiera tuvo como objetivo familiarizar al estudiantado con la información que debe constar en los presupuestos y en las facturas, así como 


\section{Conexão Letras}

las obligaciones fiscales a que deben hacer frente en el caso de facturar. También se trató el contenido de un contrato de traducción para aquellos egresados que presten sus servicios a una editorial.

\section{Visitas a entidades}

El objetivo de las visitas a entidades fue familiarizar al estudiantado con el funcionamiento de una agencia de traducción.

Este tipo de visitas permitieron que el estudiantado conociera:

- el organigrama de una agencia

- el flujo de trabajo

- las tareas que realiza cada miembro

- las herramientas usadas en cada tarea

- el tipo de textos que se traducen

- las estrategias de captación de clientes

- las competencias requeridas a sus empleados

\section{Consultorías personalizadas}

El objetivo de las consultorías personalizadas fue orientar al estudiantado en su búsqueda de empleo.

Las consultorías consistieron en atender las dudas y preguntas relacionadas con la profesión formuladas por los estudiantes, tanto personalmente como por correo electrónico. Algunas de estas consultorías se realizaron individualmente, a petición de los propios estudiantes, y otras se realizaron en grupo.

Los temas tratados fueron principalmente:

- el currículo

- las tarifas

- la facturación

- la visibilidad del traductor en diferentes portales

- las características de las pruebas de traducción

- la creación de una empresa de traducción

- la prueba de traducción

\section{Prácticas curriculares}

La asignatura de prácticas es una asignatura optativa de $4^{\circ}$ curso. Consiste en la realización de tareas relacionadas con la traducción o la interpretación en empresas, instituciones, ONG o entidades. Los estudiantes que se matriculan en esta asignatura deben hacer 75 horas de trabajo en la entidad.

El objetivo de las prácticas curriculares es:

- Fomentar el primer contacto del estudiantado con el mercado de trabajo.

- Familiarizar al estudiantado con el flujo de trabajo de una empresa o agencia de traducción.

- Potenciar el desarrollo de las competencias específicas y las competencias transversales.

- Conocer cuáles son las competencias que necesitarán en el futuro. 
Gracias a los convenios de prácticas con entidades en diferentes países de Europa, los estudiantes tuvieron la posibilidad de realizar sus prácticas en el extranjero.

Siempre que fue posible, se asignaron las plazas de prácticas para que los estudiantes pudieran trabajar con su segunda lengua extranjera. Esta decisión se tomó al considerar que su segunda lengua extranjera será el rasgo diferencial, en relación con sus compañeros, en el momento que entren a formar parte del mercado de trabajo. Esta decisión no fue bien aceptada por los estudiantes en un primer momento, ya que a menudo consideran que no tienen suficiente nivel en ese idioma; no obstante, al finalizar las prácticas la mayoría de estudiantes valoró muy positivamente haber podido trabajar con ese idioma.

Como instrumento de evaluación de las prácticas se implementó la carpeta profesional (Galán-Mañas, 2014a). A pesar de la importancia que tienen las prácticas curriculares como primer contacto con el mercado de la traducción o la interpretación, solo el 30\% de nuestros estudiantes se matriculó en la asignatura. Este dato es realmente llamativo si se tiene en cuenta que para algunas empresas esta es una cantera frecuente de candidatos (Valero, 2003).

\section{Trabajo de fin de grado}

Todos los estudios de grado en España deben concluir con la elaboración y presentación de un trabajo de fin de grado (TFG) que permita evaluar de forma global y sintética las competencias específicas y transversales asociadas al título.

En el grado de traducción e interpretación los estudiantes pueden acogerse a propuestas planteadas por los tutores o hacer ellos mismos una propuesta. Con el objetivo de promover el emprendimiento a través de los TFG, se hizo una propuesta para que los estudiantes que lo desearan realizaran un TFG relacionado con la creación de un proyecto profesional o empresarial. De este modo, podrían conocer todas las etapas de la creación de una empresa o de un proyecto profesional.

Los TFG que se elaboraron tuvieron que definir:

- las características del producto o servicio: traducción (de qué especialidad), revisión, postedición, interpretación (de qué especialidad)

- $\quad$ el tipo de necesidad que deseaban cubrir

- el proceso para prestar el servicio

- $\quad$ el segmento del mercado al que iba a ir dirigido el servicio, el perfil del cliente, el tipo de competencia

- la estrategia de desarrollo: política de nuestro servicio, precio, cómo se dará a conocer el servicio, previsión de ingresos, gastos fijos y variables

- la organización de personal, es decir, el organigrama de los puestos de trabajo, el equipo de personas necesario y las funciones que iban a desarrollar, retribuciones

- los aspectos económicos: inversión inicial necesaria, fuentes de financiación disponible, previsión de resultados a tres y cinco años

- los aspectos jurídicos: forma jurídica que adoptará el negocio, es decir, si se trataba de un proyecto profesional para el que el individuo se daría de alta como autónomo, o de un proyecto empresarial en el que el individuo crearía una sociedad limitada o una sociedad anónima. En este último supuesto, el estudiante debía especificar cuál sería el número de participantes en el proyecto empresarial y cuál sería la responsabilidad de los promotores. 
Desde las universidades, y especialmente en el área de las Humanidades, debería darse más importancia al emprendimiento. Según los datos del estudio con egresados en traducción e interpretación de la UAB mencionado en la introducción, de los egresados que llevan en el mercado entre 6 y 9 años un 3,8\% de tienen empresa propia, un 21,8\% es autónomo y un $9 \%$ trabaja a tiempo parcial en una empresa y completa su jornada laboral como autónomo. Esto significa que un 34,6 \% de los egresados de entre 6 y 9 años han tenido que crear su propio proyecto personal o empresarial muy probablemente sin formación específica alguna.

\section{Clases prácticas}

Como sostienen Calvo y Morón (2009) la filosofía de la empleabilidad también marca las metodologías docentes, ya que debe fomentar aprendizajes aplicados. La formación de traductores e intérpretes -al ser la traducción y la interpretación saberes procedimentales- siempre se ha caracterizado por el uso de metodologías activas, como el aprendizaje por tareas y proyectos, el aprendizaje basado en problemas o los estudios de caso.

El grado en traducción e interpretación de la UAB, como el resto de grados en el EEES, se ha diseñado a partir de competencias, y entre sus principales metodologías docentes destaca el aprendizaje por tareas y proyectos. Esta metodología permite que el estudiante movilice aprendizajes previos en la realización de una tarea (en los ciclos iniciales de la formación) o de un proyecto (en los ciclos finales).

Numerosos son los autores que defienden el aprendizaje por tareas y proyectos en el ámbito de la traducción y la interpretación: Hurtado (1999, 2007, 2015); González Davies (2004); Kelly (2005); Way (2008); Galán-Mañas (2007, 2009, 2013, 2014b); Stewart, Orbán y Cornellius, (2010); Fernández y Sempere (2010); Mitchell-Schuitevoerder (2011); García y Veiga (2014) y Galán-Mañas y Hurtado (2015).

Otros autores defienden el uso de textos auténticos en la realización de tareas y proyectos de traducción en el aula (Kiraly, 2000; González Davies y Kiraly, 2006; Sevilla, 2006; Galán-Mañas, 2011 y Biel, 2011). Este tipo de práctica, como sugiere Bernardini (2004), ayuda a los estudiantes a familiarizarse y a resolver problemas a los que deberán enfrentarse más tarde como profesionales.

\section{Test de competencias}

La adquisición de las competencias específicas, como apunta Pelayo (2013), es importante como factor que facilita la inserción laboral del individuo, pero no garantizan una efectiva inclusión al empleo. Lo que valoran las empresas son las diferentes habilidades - transversales- de la persona que se postula a un puesto (comunicación, trabajo en equipo, liderazgo, organización, empatía, capacidad de adaptación, iniciativa, autonomía). Para medir estas habilidades usan un test de competencias.

Para el individuo es importante disponer de esta información antes de pasar por un proceso de selección por dos razones: 1) valorar si su nivel competencial se adecua al perfil profesional que quiere alcanzar, y 2) trazar un plan de mejora, con formación específica. Además, es importante conocer las propias habilidades para elaborar algunos de los documentos esenciales para la búsqueda de empleo, como la carta de motivación, el currículo, el proyecto profesional o empresarial y la carpeta profesional.

Por ello, se recomendó a los estudiantes que realizaran el test de competencias competencies.clau, que el ayuntamiento de Barcelona pone a disposición de la población. Este test evalúa 21 competencias: iniciativa, orientación a la excelencia, planificación y 
organización, preocupación por el orden y la calidad, dirección de personas, liderazgo, trabajo en equipo y cooperación, autoconfianza, autocontrol, compromiso con la empresa, flexibilidad y gestión del cambio, etc., o el test PAPI, disponible en Treball Campus, la agencia de ocupación de la universidad, que recoge información sobre liderazgo, planificación y autonomía, capacidad de adaptación y perseverancia, búsqueda de la excelencia, trabajo en equipo, ritmo de trabajo y toma de decisiones, autocontrol, búsqueda del éxito. En ambos tests, los datos resultantes eran confidenciales y solo los alumnos tuvieron acceso a ellos.

\section{Difusión de ofertas de trabajo}

Otra de las formas de colaboración con las empresas consistió en la creación de una bolsa de trabajo con las ofertas de empleo relacionadas con la traducción o la interpretación que llegaron a la facultad. Se excluyeron aquellas ofertas que no tenían una relación directa con el plan de estudios del grado, aunque uno de los requisitos fueran los idiomas. La difusión de las ofertas se hizo a través del Consejo de Estudiantes, que publicaban las ofertas en su muro de Facebook.

\section{VALORACIÓN DEL PROGRAMA}

Las acciones previstas en el programa siguen las prescripciones del EEES y del Estatuto del Estudiante Universitario.

Entre los aspectos positivos destacamos:

- El estudiantado ha podido conocer distintas realidades del mercado local de la traducción y la interpretación de la mano de profesionales diversos: jóvenes traductores e intérpretes que se han incorporado al mercado recientemente, propietarios de agencias de traducción, traductores e intérpretes autónomos, traductores e intérpretes de las instituciones europeas, así como la diversidad de tareas realizadas en función de la especialidad.

- La variedad de acciones ha posibilitado diferentes experiencias: contacto con profesionales, contacto con empresas, elaboración de documentos para iniciar la búsqueda de empleo, conocimiento de su propio nivel de competencias transversales.

- Los talleres han sido especialmente bien valorados por los estudiantes por la actitud activa que han tenido que tener y porque les han permitido elaborar su currículo y su carta de motivación -y que se lo corrigieran-, así como otros documentos para la gestión fiscal que les servirán como modelo. También han servido para que tengan conciencia de su marca profesional y de las principales estrategias para hacerse visibles en las redes sociales profesionales.

- Las prácticas han servido para fomentar que los estudiantes tuvieran su primer contacto con el mercado de trabajo de la traducción o la interpretación a través de las prácticas curriculares y, en especial, con su segunda lengua extranjera. La segunda lengua extranjera (portugués, italiano, francés, alemán, ruso, chino, japonés, árabe) será, muy probablemente, su rasgo distintivo en relación con otros profesionales. Las prácticas curriculares, por estar entre el mundo académico y el profesional, les permiten tener una primera experiencia con ese idioma.

- Se ha fomentado el emprendimiento a través del trabajo de fin de grado, talleres y consultorías. 
No obstante, también hemos observado los siguientes problemas:

- $\quad$ Si pensamos que el tipo de formación ofrecida mediante el programa es relevante para todos los estudiantes entonces no debería ser de asistencia voluntaria, sino que sus contenidos deberían incluirse en el plan de estudios. Así se garantizaría que todos los estudiantes recibieran el mismo tipo de formación.

- Se ha conseguido ofrecer una formación de calidad a coste cero gracias a la buena voluntad de las personas, tanto del mundo académico como del mundo profesional. Sin embargo, un programa con el valor formativo que pretende tener este programa no puede depender de la buena voluntad de las personas que puedan mostrar disponibilidad y buena voluntad en un momento dado, sino que tiene que tener una garantía de continuidad por parte de la universidad.

- La difusión de las acciones ha sido inadecuada. La información relativa al programa se podía encontrar en la web de la facultad; en la web también se publicaban las actividades en forma de noticia, así como en la pantalla que hay en el vestíbulo de la facultad con este fin. Por otro lado, se enviaba un correo electrónico al Consejo de Estudiantes con toda la información de cada una de las actividades para que hicieran difusión en el Facebook de los estudiantes de la facultad. Y por último, se enviaba un correo electrónico a todo el profesorado de la facultad con la información de las actividades para que la difundieran entre sus alumnos. A pesar de todo ello, percibimos que la información no llegaba a tiempo a los alumnos, o ni siquiera llegaba.

El grado de satisfacción de las personas que han participado en el programa es bastante alto. Por otro lado, ha sido considerado como una de las fortalezas y buenas prácticas del grado en traducción e interpretación de la UAB por la Agència de Qualitat Universitària en la acreditación del grado ${ }^{2}$.

A partir de estas observaciones, podemos considerar que hay varios aspectos que mejorar:

- Dada la importancia de las prácticas curriculares como espacio en que los estudiantes pueden tener su primer contacto con el mercado y enfrentarse a plazos de entrega, textos largos, textos originales de muy poca calidad Mossop (cit. Durban et al. 2003), habría que considerar la obligatoriedad de las prácticas curriculares en el plan de estudios.

- Se debería impulsar el contacto directo con profesionales dentro del aula en la última fase de formación, de este modo se estimularía el interés por la profesión y el conocimiento de los requerimientos de la profesión: plazos, tipos de clientes, tipos de textos, flujo de trabajo, control de calidad, etc. El hecho de poder conocer de primera mano el mercado, acompañados por profesionales, también favorecería que en el momento en que el individuo vaya a buscar trabajo haya adquirido cierta madurez profesional y, en consecuencia, autoestima profesional, un aspecto que raramente se tiene en cuenta en la formación de traductores.

- Como hemos mencionado, los resultados de los test de competencias solo los reci-

2 http://www.uab.cat/doc/InformeAcreditacioGrauTraduccioInterpretacio2014 
bían los estudiantes. Se trabajó con Barcelona Activa para que la facultad pudiera acceder a los resultados anónimos, pero agregados, sin embargo, aún no ha sido posible. Esto permitiría identificar carencias en alguna competencia transversal, pero valorada por las empresas de traducción e interpretación y que pudiera organizar algún taller, especialmente entre los estudiantes de tercero y cuarto.

- Habría que mejorar la difusión de las actividades y posiblemente buscar otros canales de difusión que no se han explotado, como Twitter.

\section{Conclusiones}

El programa de formación para la empleabilidad de los traductores e intérpretes de la Universitat Autònoma de Barcelona ha servido para fomentar la colaboración entre la universidad y los profesionales y las empresas del entorno. Una vez implementado el programa, es necesario evaluar la empleabilidad de las personas que lo han seguido. Sin embargo, como sostiene Calvo (2013), eso es algo muy difícil.

Dada esta dificultad, acabamos midiendo la inserción laboral de los egresados en traducción e interpretación, es decir, qué número de egresados tiene trabajo un cierto tiempo después de finalizar los estudios. En el ámbito de la traducción y la interpretación, incluso este dato es especialmente difícil de recoger, ya que, por un lado, muchos egresados realizan tareas diversas, algunas de las cuales ni siquiera están relacionadas con la traducción o la interpretación y, por otro lado, en los primeros momentos después del egreso hacen trabajos precarios para los que ni siquiera están dados de alta como autónomos. En este sentido, ¿podemos considerar que una persona está insertada si realiza traducciones muy esporádicamente, aunque no consiga ganarse el sustento con la traducción o la interpretación? Sería importante, pues, tener en cuenta cuál es la principal fuente de ingresos de los egresados para identificar su grado de relación con el mercado de trabajo.

Ahora bien, la empleabilidad no debería ser exclusivamente un indicador cuantitativo, sino también (y sobre todo) un indicador cualitativo (Calvo, 2013). Ello nos ayudaría a tener en cuenta en los estudios de inserción laboral los aspectos que enumera la autora y que ciertamente condicionan la inserción laboral del individuo: clase social, género, raza, discapacidad, edad, hijos a su cargo, reputación de la universidad en la que ha estudiado, situación económica del mercado, experiencia laboral previa, existencia de un programa de orientación profesional durante su formación.

Pensamos que el programa para la mejora de la empleabilidad de los egresados en Traducción e Interpretación que hemos presentado tiene en cuenta las recomendaciones del Estatuto del Estudiante Universitario, según el cual el estudiantado tiene derecho a recibir orientación y tutoría en la fase final de la formación. El contenido del programa también contempla las recomendaciones del Estatuto, ya que incluye formación en itinerarios formativos y salidas profesionales, ayuda al estudiante a identificar sus propias competencias y le orienta en el diseño de su proyecto profesional para facilitarle su empleabilidad y su inserción laboral.

El programa está abierto a todo el estudiantado, de modo que aquellos individuos de los niveles iniciales que se sientan preparados para diseñar su proyecto profesional y buscar empleo, tendrán la oportunidad de hacerlo.

Por último, es importante resaltar que el programa no solo pretende asegurar la inserción laboral de los egresados, sino también la calidad de su inserción. 


\section{Bibliografía}

AGUESSIM, Abdellatif. El mercado profesional en traducción jurídica y jurada y en mediación intercultural: El caso de la lengua árabe». Tonos Digital. Revista Electrónica de Estudios Filológicos, 15, 2008.

AGUILAR, José Miguel. La enseñanza de la traducción y su relación con las exigencias del mercado laboral: estudio de caso. Trans. Revista de Traductología, 8: 11-28, 2004. AHMED, Elimam. The Impact of Translation Memory Tools on the Translation Profession. The Translation Journal, 11, 2007.

ALCINA, Amparo. Encouraging the use of e-mail and mailing lists among translation students. Meta. Translators' Journal 48(4), 634-641, 2003.

AMIT-KOCHAVI, Hanna. The people behind the words: Professional profiles and activity patterns of translators of Arabic literature into Hebrew (1896-2009). TIS-Translation and Interpreting Studies, 5:1, 41-58, 2010.

ANECA. Libro blanco. Título de grado en Traducción e Interpretación. Madrid: Agencia Nacional de Evaluación de la Calidad y Acreditación, 2005.

ANGELELLI, Claudia. A professional ideology in the making: Bilingual youngsters interpreting for their communities and the notion of (no) choice. TIS (Translation and Interpreting Studies), 5:1, 94-108, 2010.

BADIA, Toni. Integració de la TA en el procés professional de la traducció. Revista Tradumàtica. Traducció i tecnologies de la informació i la comunicació, 4, 2006. BERNARDINI, Silvia. The Theory Behind the Practice: Translator Training or Translator Education?. En Kirsten Malmkjaer (ed.) Translation in Undergraduate Degree Programmes. Amsterdam and Philadelphia: John Benjamins, 17-31, 2004.

BIEL, Łucja. Professional Realism in the Legal Translation Classroom: Translation Competence and Translator Competence. Meta. Translators' Journal, 56(1), 162-178, 2011.

BIELSA, Esperança. Translation in global news. Target, 19(1): 135-155, 2007. BISQUERRA, Rafael. Orientació psicopedagògica i desenvolupament de recursoshumans. Barcelona: La Llar del Llibre, 1992a.

BOWKER, Lynne. What Does It Take to Work in the Translation Profession in Canada in the 21st Century? Exploring a Database of Job Advertisements. Meta. Translators' Journal, 49(4): 960-972, 2004.

CALVO, Elisa. Análisis de la empleabilidad como indicador de la calidad universitaria: ¿qué nos enseñan las experiencias anglosajonas?. En: Alberti, Giorgio et al. Desafios y oportunidades de la empleabilidad de los egresados universitarios en el contexto internacional. Madrid: McGraw-Hill/Intermaricana de España, 2013.

. Orientación Profesional para Futuros Licenciados de Traducción e Interpretación: Estrategias Centradas en las Necesidades del Estudiantado. En S. Bravo Utrera y R. García López (eds.), Estudios de Traducción: Problemas y Perspectivas. Las Palmas de Gran Canaria: Servicio de Publicaciones de la Universidad de Las Palmas. 633-649, 2006.

CALVO, Elisa y MORÓN, Marian. Tutorías para la empleabilidad en la FTI de la UGR. Seminario Internacional: El desarrollo de la autonomía en el aprendizaje (2/7/2009), Red Estatal de Docencia Universitaria (REDU), 2009.

CALVO, Elisa et al. A Project to Boost and Improve Employability Chances among 
Translation and Interpreting Graduates in Spain. En Valerie Pellatt et al. (eds.) Teaching and Testing Interpreting and Translating. Intercultural Studies and Foreign Language Learning. Vol. 2. Berlín: Peter Lang, 2010.

CEREZO, Beatriz. La didáctica de la traducción audiovisual en España: Un estudio de caso empírico-descriptivo. Tesis doctoral. Castelló de la Plana: Universitat Jaume I. 2012. CHAUME, Frederic. The turn of audiovisual translation: New audiences and new technologies. Translation Spaces, 2, 107-125, 2013.

CHOUC, Fanny y CALVO, Elisa. Embedding employability in the curriculum and building bridges between academia and the work-place: a critical analysis of two approaches. La linterna del traductor, 4: 71-86, 2010.

CHRUPALA, Grzegorz. Perl Scripting in Translation Project Management. Across Languages and Cultures, 4:1, 2003.

COLLET, Thaís. O mercado de tradução audiovisual no Brasil: formação e demanda. Tesis doctoral. Florianópolis: Universidade Federal de Santa Catarina, 2016.

DAM, Helle y ZETHSEN, Karen Korning. Translators in international organizations: A special breed of high-status professionals? Danish EU translators as a case in point. Translation and Interpreting Studies, 7(2), 2012.

DEL POZO, Rocío La integración de los dispositivos móviles en el entorno de trabajo de los profesionales de la traducción y la interpretación. Revista Tradumática, 14, 2016. DÍAZ CINTAS, Jorge. Teoría y práctica de la subtitulación inglés-español. Barcelona: Ariel, 2003.

DURBAN, Chris et al. Translator Training and the Real World: Concrete Suggestions for Bridging the Gap. Round Table. Part A, Translation Journal, 7(1), 2003. Disponible en: http://www.bokorlang.com/journal/23roundtablea.htm.

FERNÁNDEZ, Chus y SEMPERE, Francisca. Shifting from translation competence to translator competence. En Valerie Pellatt, Kate Griffiths y Wo Shao-Chuan (Eds.), Teaching and testing interpreting and translating (p. 131-148). Oxford: Peter Lang, 2010. FLORIDO, Isabel. Un Ensayo sobre Nuevas Salidas Profesionales Nuevas Exigencias de Formación y Especialización. Revista de lenguas para fines específicos, 2, 1995. GALÁN-MAÑ̃S, Anabel. Vinculación de las prácticas curriculares con la realidad laboral de los graduados en Traducción e Interpretación. VIII Congreso Internacional de la Asociación Ibérica de Estudios de Traducción e Interpretación. Alcalá de Henares, 8-10 de marzo de 2017.

. Enseñanza de la Iniciación a la traducción basada en competencias: Resultados de una validación empírica. Revista Universitas Tarraconensis. Vol. 2, desembre 2014, pp. 27-47, 2014b.

. The professional portfolio as an instrument to assess internship. 14th Annual Portsmouth Translation Conference: From classroom to workplace. Portsmouth, Inglaterra, (8/11/2014), 2014a.

. L'apprentissage par projets dans la formation de traducteurs. Une expérience pour professionnaliser l'étudiant. Babel. Revue de Traduction. Vol. 59 (1), pp. 41-56, 2013.

. Translating authentic technical documents in specialised translation classes. Journal of Specialized Translation (Jostrans), 16, 2011.

. La enseñanza de la traducción en la modalidad semipresencial. Tesis doctoral. Bellaterra: Universitat Autònoma de Barcelona, 2009.

. La enseñanza por competencias, por tareas y por objetivos de aprendizaje: el caso de la traducción jurídica portugués-español. ÍKALA. Revista de lenguaje y cultura, Vol. 12 (18), pp. 25-58, 2007. 


\section{Conexão Letras}

GALÁN-MAÑAS, Anabel y HURTADO, Amparo. Competence assessment procedures in translator training, The Interpreter and Translator Trainer, 9(1), 63-82, 2015.

GARCÍA, Marta; VEIGA, María Teresa. Guided Inquiry and Project-Based Learning in the field of specialised translation: a description of two learning experiences. Perspectives Studies in Translatology, 2014.

GARCÍA, Juan Vicent y PÉREZ, Mari Carmen. Espacio Europeo de Educación Superior, competencias profesionales y empleabilidad. Revista Iberoamericana de Educación, 46, 2008.

GONZÁLEZ DAVIES, Maria; KIRALY, Don. Translation: Pedagogy. Encyclopedia of Language and Linguistics, 14 (vol. set.), pp.81-85, 2006.

GONZÁLEZ DAVIES, María. Multiple voices in the translation classroom: activities, tasks and projects. Amsterdam: John Benjamins, 2004.

GOUADEC, Daniel. Translation as a profession, Amsterdam y Philadelphia: John Benjamins, 2007.

HILLAGE, James y POLLARD, Emma. Employability: developing a framework for policy analysis. Research Brief 85, Department for Education and Employment, 1998. HOLMES, James. The Name and Nature of Translation Studies, Tranlsated. Papers in Literary Transaltion and Translation Stadies. Amsterdam: Rodopi, 1972/1988.

HURTADO, Amparo. Aprender a traducir del francés al español. Competencias y tareas para la iniciación a la traducción. Serie Aprender a traducir, Edelsa - Universitat Jaume I, 2015.

. Competence-based curriculum design for training translators. The Interpreter and Translator Trainer, 1(2), 163-195, 2007.

HURTADO, Amparo (dir.) Enseñar a traducir. Metodología en la formación de traductores e intérpretes. Madrid: Edelsa, 1999.

ICE. Competencias genéricas y transversales de los titulados universitarios. Zaragoza: Universidad de Zaragoza, 2008.

JIMÉNEZ, Óscar. La traducción técnica inglés-español: didáctica y mundo profesional. Granada: Comares, 2002.

KAGEURA, Kyo et al. Has translation gone online and collaborative?: An experience from Minna no Hon'yaku. Linguistica Antverpiensia, New Series - Themes in Translation Studies, 10, 2011.

KELLY, Dorothy. A Handbook for Translator Trainers. A Guide to Reflective Practice. Manchester: St Jerome Publishing, 2005.

. La investigación sobre formación de traductores: algunas reflexiones y propuestas. En: Emilio Ortega (dir.) Panorama actual de la investigación en Traducción e Interpretación 1, Granada: Atrio, 585-596, 2003.

KELLY, Nataly; RAY, Rebecca; DEPALMA, Donald. From crawling to sprinting: Community translation goes mainstream. Linguistica Anterpiensia, New Series-Themes in Translation Studies, 10, 2011.

KIRALY, Don. Project-based Learning: A case for situated translation, Meta. Translators' Journal, 50(4), 1098-1111, 2005.

. A Social constructivist approach to translator education: empowerment from theory to practice. Manchester: St. Jerome, 2000.

KLIMKOWSKI, Konrad. Team work as a method in translation. Across Languages and Cultures, 7 (1), 2006. 
KUZNIK, Anna. Puestos de trabajo híbridos. Cuatro indicadores del carácter heterogéneo de los puestos de trabajo internos en traducción. Sendebar, 22, 2011.

. El contenido de los puestos de trabajo de los traductores. El caso de los traductores internos en las empresas de traducción de Barcelona. Tesis doctoral. Bellaterra: Universidad Autónoma de Barcelona, 2010.

. Marco conceptual y metodológico para un estudio sobre las tareas de traducción e interpretación desempeñadas en el ámbito laboral. Trabajo de investigación de doctorado. Bellaterra: Universidad Autónoma de Barcelona, 2007.

LEBTAHI, Yannicke e IBERT, Jérôme. Traducters dans la société de l'information. Evolutions et interdépendances. Meta. Translators' Journal, 49(2), 221-235, 2004. KO, Leong. Translation checking: a view from the translation market. Perspectives: Studies in Translatology, 19(2), 123-134, 2011.

LI, Defeng. Translator Training: What Translation Students Have to Say. Meta: Translators' Journal, 47(4): 513-531, 2002.

. Translator Training in Hong Kong: What Professional Translators Can Tell Us.

En: S. Chan (ed.) Translation in Hong Kong: Past, Present and Future. Hong Kong: The Chinese University Press, 85-96, 2001.

MAYOR SERRANO, Blanca. ¿Y ahora qué? Salidas profesionales del traductor médico. Panacea: boletín de medicina y traducción, 5 (15), 2005.

MAYR, Eva et al. Organisational Analysis as Important Factor for Optimization of Workplace Learning. En: Sibille HAMBACH, Alke MARTENS y Bodo URBAN (eds.) eLearning Baltics 2008: Proceedings of the 1st International eLBa Science Conference, Rostock, Alemania (18-19/6/2008). Stuttgart: Fraunhofer IRB, 1-10, 2008.

MIKKELSON, Holly. The Critical Link: Professionalisation of interpreting in the community. Selected papers from the 4th International Conference on Interpreting in Legal, Health and Social Service Settings. Estocolmo, Suecia. (Mayo 2004). Benjamins Translation Library, 2007.

MINISTERIO DE EDUCACIÓN DE ESPAÑA (2010). Estatuto del estudiante universitario (2011), Real Decreto 1791/2010, de 30 de diciembre.

MITCHELL-SCHUITEVOERDER, Rosemary. Translation and technology in a project based learning environment. Session 3 - Training translators / La formation du traducteur, 2011. Disponible en: http://lodel.irevues.inist.fr/tralogy/index.php?id=173.

MONZÓ, Ester. La professió del traductor jurídic i jurat: descripció sociològica del professional i anàlisi discursiva del transgènere. Tesis doctoral. Castelló de la Plana: Universitat Jaume I, 2002.

MORRIS, Ruth. La interpretación en el Tribunal de Justicia 2009: Una profesión infravalorada e incomprendida? O bien: la justicia hablará?. MonTI, 2, 2010.

ORDÓÑEZ, Pilar. De mayor quiero ser traductor I. Estudio de las preconcepciones de los alumnos de primer curso del Grado en Traducción e Interpretación. inTRAlinea, 12, 2010 .

ORF, Dorothee. Estudio de situación del mercado español de servicios profesionales de traducción. Madrid: Agrupación de Centros Especializados en Traducción, 2005.

ORTEGA, Juan Miguel. Cómo acreditar intérpretes a través de la traducción: análisis crítico de la acreditación profesional de intérpretes jurídicos en España. Trans. Revista de Traductología, 2011. 


\section{Conexão Letras}

PELAYO, Mariana Betzabeth. Capital social y competencias profesionales: Factores condicionantes para la inserción laboral, 2013. Disponible en http://www.eumed.net/. PIRCHER, Richard et al. Organizational Learning and Knowledge Transfer in Expanding Companies - Findings from an Empirical Study on Austrian Companies Entering Eastern European Markets. En: Sivia Gherardi y Davide Nicolini (eds). The Passion for Learning and Knowing. Proceedings of the 6th International Conference on Organizational, 2005. POLLEY, Cameron y BARBER, Paul. Translation as a business. The translator's handbook, London: Aslib, 1989.

PYM, Anthony et al. Work placements in doctoral research training in the humanities:

Eight cases from translation studies. Across Languages and Cultures, 15(1), 1-23, 2014. Real Decreto 1393/2007 de 29 de octubre, por el que se establece la ordenación de las enseñanzas universitarias oficiales. BOE núm. 260 del martes 30 de octubre de 2007. Pp. 44037- 44048.

ROMERO, Virginia. Inserción Ocupacional. Barcelona: Altamar, 2004.

SALES, Dora; VALERO, Carmen. Bibliografía sobre traducción e interpretación en los servicios públicos y mediación intercultural. Resla, Revista de Lingüística Aplicada. Volumen monográfico, 2006.

SAMUELSSON-BROWN, Geoffrey. A practical guide for translators. Clevedon: Multilingual Matters, 1998.

SCHELLEKENS, Philida. Workforce Research: Interpreting and Translation: Key Findings and Recommendations. FCO Services and CILT, 2004.

SEVILLA, Manuel. Analysis of the Pedagogic Potential of a Text as Didactic Material for the Subject of Scientific and Technical Translation. Didáctica (Lengua y Literatura), 18, 295-314, 2006.

SOFER, Morry. The translator's handbook. Washington: Schreiber, 2004.

STEWART, John; ORBÁN, Wenke y KORNELIUS, Joachim. Cooperative learning in the paradigm of project based learning. T21N Translation in Transition, 1, 1-28, 2010. TEICHMAN, Diane. Best face forward: In person marketing skills for T\&I professionals, 2003. Disponible en: http://www.lexicool.com.

TORRES-HOSTENCH, Olga. L'ús de tecnologies en les pràctiques de traducció en empresa. Tradumàtica, Revista Tradumàtica: tecnologies de la traducció, 11, 286-296, 2013.

. An overview of the research on occupational integration in translation studies according to a map of occupational integration concepts. Perspectives: Studies in Translatology 18:1, 2010.

. Programa de formació per a la inserció laboral dels postgraduats en traducció. Tesi doctoral. Bellaterra: Universitat Autònoma de Barcelona, 2007.

ULRYCH, Margherita. Real-world criteria in translation pedagogy. Teaching translation and interpreting, 3, 251 259, 1996.

VALERO, Carmen. Traducción e interpretación en los servicios públicos: Contextualización, actualidad y futuro, Granada: Comares, 2003.

WAGNER, Emma; BENCH, Svend y MARTÍNEZ, Jesús. Translating for the European Union Institution, Manchester: St. Jerome, 2002.

WAKABAYASHI, Judy. Induction into the translation profession through Internet mailing lists for translators. En Teaching translation and interpreting 4. Building bridges, Edited by: Hung, E. 47-58. Amsterdam: John Benjamins, 2002. 
WAY, Cathy. Eighth Portsmouth Translation Conference: The Changing Face of translation. Eighth Portsmouth Translation Conference: The Changing Face of translation. Portsmouth University, Reino Unido, 2008.

Recebido em: 25/04/2017. Aceito em: 04/05/2017 